\title{
6 Das Echo der Vergangenheit: „Der freche Jude hetzt wieder gegen Deutsche!“
}

\subsection{Versatzstücke der NS-Sprache im judenfeindlichen Gegenwartsdiskurs}

Wir haben gezeigt, dass sich im antisemitischen Diskurs der Gegenwart oft Tendenzen der Schuld- und Erinnerungsabwehr in Bezug auf die deutsche Geschichte $^{1}$ zeigen. Viele Sprachproduzenten kommunizieren dabei explizit oder implizit judenfeindliche Stereotype, die frequent in der NS-Zeit artikuliert wurden. Zudem lässt ihre Argumentation, die vergangenheitsbezogene Aspekte und gegenwartsbezogene Themen vermischt, erkennen, wie präsent und wichtig die Vergangenheitsbewältigung auch über 60 Jahre nach dem Zusammenbruch des nationalsozialistischen Gewaltregimes noch immer ist. In diesem Kapitel soll gezeigt werden, inwieweit konkrete Wörter und Phrasen, die in der NS-Zeit häufig benutzt wurden, noch heute in judenfeindlichen Texten reproduziert ${ }^{2}$ werden und welche Auswirkungen diese Sprachgebrauchsmuster auf den aktuellen Verbal-Antisemitismus haben. Finden sich also neben den bloßen Reproduktionen von Versatzstücken aus der NS-Ideologiesprache Hinweise dafür, dass auch

1 Wir können also nicht die in Teilen der Antisemitismusforschung vertretene These bestätigen (vgl. u. a. Rabinovici et al. 2004: 8), dass die Vergangenheitsbewältigung im aktuellen Antisemitismus immer mehr an Einfluss verliere und stattdessen antizionistische und anti-israelische Tendenzen überwiegen. Vielmehr vermischen sich vergangenheitsund gegenwartsbezogene Argumente in aktuellen antisemitischen Texten. Die kontrastive Korpusanalyse zu Texten aus anderen europäischen Ländern zeigt, dass dies jedoch in besonderem Maße auf Deutschland zutrifft. In anderen europäischen Ländern sind tatsächlich Formen des Anti-Israelismus und dabei Kombinationen von klassischen judeophoben und aktuellen antizionistischen Klischees dominant, während die Schuldthematisierung keine bzw. nur eine marginale Rolle spielt (s. Kap. 8).

2 Eitz/Stötzel (2007 und 2009) haben anhand von Stichwort-Analysen zu ca. 50 „NSVokabeln“ untersucht, inwieweit Lexeme aus der NS-Zeit im aktuellen öffentlichen Diskurs teils intentional-persuasiv, teils unreflektiert Verwendung finden. Insbesondere NS-Vergleiche werden dieser Analyse zufolge seit der Jahrtausendwende geradezu inflationär benutzt. Dabei werden insbesondere die Wörter Auschwitz, Holocaust und Endlösung hochfrequent verwendet (vgl. Eitz/Stötzel 2007: 175), was exakt den Befunden entspricht, die wir im Korpus registriert haben. NS-Vergleiche dienen im massenmedialen Raum vor allem der Intensivierung einer Beleidigung oder Polemik (vgl. Eitz/Stötzel 2007: 304 f.). Die Mehrzahl der NS-Vergleiche sind allerdings keineswegs verbale Ausrutscher oder Entgleisungen, sondern mit Kalkül ausgewählte Kommunikationsmuster (s. hierzu Schwarz-Friesel 2007: 197). NS-Vergleiche in judenfeindlichen Äußerungen dienen der Stigmatisierung und Diffamierung.

DOI 10.1515/9783110277722.174, (c) BY-NC-ND , published by De Gruyter.

This work is licensed under the Creative Commons Attribution-NonCommercial-NoDerivs 4.0 License. 
semantisch-konzeptuelle Argumente und Begründungszusammenhänge übernommen werden?

Die sprachlichen Kennzeichen und kommunikativen Funktionen des Antisemitismus in der NS-Zeit sind bereits in vielen philologischen und linguistischen Ansätzen intensiv untersucht und beschrieben worden (vgl. u. a. die LTI Klemperers [1947] 242010 sowie Ehlich 1989, Bering 1991 und ${ }^{3} 1991$, Kinne/Schwitalla 1994, Schmitz-Berning 1998, Hortzitz 1999, Hutton 1999, Braun 2007).

Die wesentlichen Charakteristika ${ }^{3}$ der Sprachgebrauchsmuster sind: Stigmatisierung durch Namenszuordnung (Sarah, Israel, Cohen, Itzig), Veränderung von Appellativa, d.h. Berufsbezeichnungen als pejorative Beschimpfungen (Rechtsverdreher, Zähnejuden), rassistische Metaphern durch Attributsbezeichnungen aus der Biologie (Bazillen, Blutsauger, Parasiten), Komposita mit Tierbezeichnungen (Judensau, Judenschwein), Betonung der Gattung durch generische Nominalphrasen mittels typisierendem Singular (Der Jude, Der freche Jud), Wiederholung klischeehafter Floskeln mit verschwörungstheoretischer Semantik (Weltjudentum, Verjudung, Finanzjudentum); referenzielle Reduzierung von allen Juden auf Kollektivattribute wie Geldschacherer, Krämerseele, arroganter Intellektueller, Intrigant, Bolschewik sowie generische Allaussagen und Kollektiventwertungen in Form von Phrasen und Sätzen (jüdische Greuelpropaganda, typisch jüdische Frechheit, verjudete Presse, jüdische Hetze, Juden sind unser Unglück). Alle diese Verbalisierungsformen sind Ausdruck der Ideologie der Nationalsozialisten, die auf völkisch-nationalistischen und rassistischen Konzepten basierte.

Aggressive Sprüche mit judenfeindlichen Aufforderungen, Warnungen, Verfluchungen und Drohungen wie „Deutsche, wehrt euch, kauft nicht bei Juden!“ (Berlin, 1933, vgl. Königseder 2011: 61), „Juda verrecke“ (Berlin, 1933), „Keiner soll hungern, keiner soll frieren, aber die Juden sollen krepieren“ (Deutschland, 1935, vgl. Améry [1966] 62008: 135) waren zudem zwischen 1933 und 1945 an der Tagesordnung. Alle Massenmedien ${ }^{4}$ verbreiteten judenfeindliche Propaganda, die im

3 Mit der Phrase Sprache des Nationalsozialismus sind ein typisches Vokabular und bestimmte rhetorische Strategien gemeint, die in der Zeit des Nationalsozialismus häufig verwendet wurden. Als typische lexikalische Charakteristika gelten die Verwendung nationalistischer Schlagwörter (Volk, Vaterland), biologisch-pathologischer Metaphern (Schädling) und Lexeme aus dem radikalen Rassismus (Arier, entartet, Überfremdung usw.; s. Cobet 1973: 238, SchmitzBerning 1998, von Polenz 1999: 541 ff.). Im wissenschaftlichen Sinne ist dies keine eigene Sprache, daher spricht z. B. Braun (2007) vom „nationalsozialistischen Stil“.

4 Braun (2007) erörtert neben rhetorischen und stilistischen Eigenschaften des nationalsozialistischen Sprachgebrauchs auch die Rolle der Medien bei der Verbreitung der ideologischen Semantik und der typischen Floskeln. Neben Hitler lieferten vor allem auch NSGrößen wie Goebbels und Rosenberg Vorlagen für einen gruppenspezifischen Sprachstil. 
offiziellen und öffentlichen Sprachgebrauch ${ }^{5}$ usuell wurde und entsprechend ihre Wirkung entfalten konnte.

Wir haben uns bei der Korpusanalyse auf die Lexeme (und typischen Abkürzungen) arisch, Ausmerzung, Endlösung, entartet/Entartung, Führer, Gaskammern, Gestapo(methoden), gleichschalten, Herrenrasse, Juden(frage/problem), Konzentrationslager/KZ, Propaganda, Rasse, Reichskristallnacht/Reichspogromnacht, SA, Sonderbehandlung, SS, Untermenschen, Volk(sschädlinge), Weltjudentum, zersetzen konzentriert sowie untersucht, ob Eigennamen der Nazi-Größen wie Hitler und Goebbels benutzt werden. Des Weiteren haben wir, nachdem uns in Stichprobenanalysen aufgefallen war, dass die in den Reden und Schriften Hitlers und Goebbels öfter benutzten Phrasen freche(r) Jude(n) und jüdische Hetze auch im Korpus zu beobachten sind, überprüft, inwieweit im modernen Diskurs solche für die NS-Ideologie typischen Kollokationen ${ }^{6}$ ebenfalls auftauchen.

Dass als Neonazis und Rechtsradikale identifizierte Schreiber auf verbale Mittel und Strukturen aus der NS-Zeit zurückgreifen, verwundert nicht, da diese die Ideologie dieser Phase gutheißen und sich damit identifizieren. Entsprechend ist die Verwendung von Nazi-Vokabeln ein typisches Kennzeichen der rechtsextremen Kommunikationsmuster. 98 Prozent der E-Mails und Briefe aus dem rechten Milieu enthalten daher Wörter, Phrasen oder Sätze, die in der NS-Zeit entweder zur Diffamierung von Juden oder allgemein zu Propagandazwecken und Identitätsstabilisierung benutzt wurden.

Klare Indikatoren für rechtsextreme Zuschriften sind insgesamt Grußformeln aus der NS-Zeit (z. B. Sieg Heil! oder mit germanischem Gruß, mit deutschem Gruße):

„Heil Olmert dem Fuehrer und Moerder des israelischen Verbrecherstaates.“ [ZJD_12.04.2007_Mon_001]

5 Rassistisches Gedankengut wurde im April 1933 durch die Aufnahme von Wörtern wie arisch, Ariernachweis und Arierparagraph Bestandteil des offiziellen, juristischen Diskurses (s. auch von Polenz 1999). Die Entwicklung des Rassismus hat nach Römer (1985) gezeigt, dass radikalnationalistische und besonders extrem rassistische Tendenzen auch in akademischen Kreisen, z. B. besonders stark in der (indo)germanistischen Sprachwissenschaft bereits seit der Mitte des 19. Jahrhunderts artikuliert und etabliert wurden.

6 Als Kollokationen bezeichnet man typische Wortverbindungen, also sprachliche Einheiten, die in verschiedenen Kontexten vermehrt gemeinsam auftreten. Mittels sogenannter Kookkurrenz- oder Kollokationsanalysen untersucht die Linguistik, ob und inwieweit die Wörter solcher Kollokationen in einer engen semantischen Beziehung zueinander stehen und in welchen Kontexten sie bevorzugt benutzt werden (vgl. Kap. 2.1). 
Verfasser aus der rechten Szene benutzen zudem auffällige E-Mail-Adressen mit intertextuellen Verweisen auf Nazi-Größen oder Ideologiefragmente (karinhall...@ web.de, ${ }^{7}$ wotan...@web.de), NS-Vergleichen (holocaustgaza@...com) oder unterschreiben mit Phrasen wie ein aufrichtiger Deutscher oder ein richtiger Arier. Auch verkürzte Schreibungen des Lexems Jude (z. B. der Jud Scharon in Anlehnung an Jud Süß), Einzellexeme wie Reichshauptstadt oder Ironisierungen wie sogenannte 'BRD', Schlagwörter des Nationalsozialismus (wie Volk, Reich usw.), der extensive Gebrauch des Attributs deutsch (wie in deutsche Interessen, deutsches Leid) sowie stark rassistische Vokabeln (wie Parasitenpack, Rasse, Rassenschande, Untermenschen und Untermenschengesindel) sind frequent. Von den dehumanisierenden Komposita, die in der NS-Zeit im Alltagsdiskurs viel verwendet wurden, sind Judensau und Judenschweine im Korpus besonders oft zu finden. Die Verwendung der verbalen Versatzstücke aus der nationalsozialistischen Propaganda geht mit der mentalen Ideologie einher.

Entsprechend zeichnen sich die E-Mails von Schreibern aus dem rechten Spektrum meist dadurch aus, dass Hitler und andere Nazi-Größen als „Autoritäten für das Judenproblem“ zitiert und Teile aus NS-Diskursen (Lexeme, Phrasen, Sätze) wortwörtlich reproduziert werden, um antisemitisches Gedankengut auszudrücken. Insbesondere finden sich viele Verweise auf die rassistische PseudoTheorie der Nationalsozialisten:

(2) „Dreckiges deutsches nicht arisches Illuminatenpack, nicht christlich“ [ZJD_24.05.2007_ano_001]

$$
\text { „Heil Hitler! Ihr Untermenschen!“ [IBD_07.09.2005_ano_Postkarte] }
$$

In der NS-Zeit häufig benutzte Lexeme aus Texten der „Rasselehre“ des 19. Jahrhunderts wie arisch, Rasse, minderwertig und Untermenschen werden in nahezu allen E-Mails und Briefen von Rechtsextremisten benutzt, es finden sich auch Anleihen an Sprachmuster, die in den Nürnberger Rassegesetzen festgelegt waren, wie z. B. das Kompositum Halbjude:

(4) „Macht Gregor Gysi sowas oder Reich-Ranicki? Sie sind auch Halbjude bzw. Jude und niemand nimmt daran Anstoß!!!!!!“

[ZJD_28.05.2008_Wil_001]

7 Carinhall war die von Göring gewählte Bezeichnung für ein großes Anwesen in Brandenburg (zu Ehren seiner ersten Frau Carin). 
Die Idee, Juden seien eine „Rasse für sich“, wird zum Teil aber auch von Schreibern kodiert, die keine rechtsextreme Auffassung erkennen lassen wie in (5), der E-Mail eines „einfach deutschen Bürgers aus Köln“, der sich „mitten in der Gesellschaft“ positioniert und die „Verbrechen der Nazis scharf verurteilt“:

„Meine Scham als Deutscher hinsichtlich der Verbrechen meines Volkes an Ihrer Rasse [...]“ [IBD_24.03.2009_Wah_001]

Bei solchen Verbalisierungen spielen nicht die ideologische Ausrichtung, sondern vielmehr Unkenntnis und mangelnde Bildung eine Rolle. Im Gedächtnis solcher Sprachproduzenten sind Juden konzeptualisiert als JUDEN SIND EINE RASSE (s. hierzu auch die Beispiele (80) bis (82) in Kap. 5.2).

Bei akademisch gebildeten Verfassern finden sich zwar auch NS-Lexeme aus dem Rassismus-Vokabular ${ }^{8}$ (insbesondere in NS-Vergleichen, die Israel stigmatisieren), doch so gut wie nie dienen diese dem Ausdruck einer auch konzeptuell rassistischen Argumentation oder Weltsicht. Im Gegenteil: Diese wird strikt abgelehnt, was allerdings in Widerspruch zur Verwendung von Versatzstücken aus der NS-Sprache steht (s. Kap. 5).

Hitler wird von rechtsextremen Schreibern zumeist als „großer Führer" und als „weitblickend“ bezeichnet, der „wusste, warum er die Juden ausrotten wollte“ (wobei oft Bedauern ausgedrückt wird, dass die „Ausrottung nicht zum Ende gebracht wurde"). Gegenwartsbezogen finden sich Dehumanisierungen und Dämonisierungen der heute lebenden Juden und/oder Israelis mittels NS-Lexik und Metaphorik (wobei Tier-, Schädlings- und Krankheitsmetaphern dominieren; vgl. auch Kap. 10).

$$
\text { „Parasitenpack, dreckige Judenschweine.“ [ZJD_29.03.2009_Zel_002] }
$$

„Schädlinge! Teufelsbrut! Anti-Christen, Krebsgeschwür des nahen Osten.“ [IBD_07.05.2010_ano_003]

Entsprechend stellen die Verfasser die „Vollendung der Endlösung“ mittels Drohungen, Wünschen und Prophezeiungen in Aussicht (s. hierzu auch Kap. 10.1):

8 Der Begriff „Rasse“ taucht allerdings auch heute noch (oft ohne kritische Distanzierungsmerkmale) in wissenschaftlichen Abhandlungen auf. In vielen Besprechungen zu Hausarbeiten und Thesenpapieren von Studierenden zu Verbal-Antisemitismus und Rassismus muss zudem regelmäßig darauf hingewiesen werden, dass dieser Begriff obsolet ist, weil die Hypothese von Rassen wissenschaftlich in der Biologie widerlegt ist, und durch „Ethnie“ zu ersetzen. 
(8) „Wird wieder einmnal Zeit das richtige Arier den Gashahn aufdrehen! [...] HEIL HITLER!“ [IBD_19.01.2004_ano_001] „Abmarsch in die Gaskammer!“ [ZJD_Gaza2009_664/816_Pan_001]

Anti-israelische Dämonisierungen und judeophobe verschwörungstheoretische Konzepte werden dabei argumentativ besonders häufig miteinander verbunden.
„Ihr Palästinenser-Killer!...zur naechsten 'Kristallnacht' diesmal werden wir kein Glas zerbrechen, sondern nur eure dummen mit Voelkermord- scheisse gefuellten Schaedel zerbrechen. . Die 'Kristallnacht' wird ein weltweites Ereignis werden! Denn wir wollen uns endlich vom Joch und den Spinnereien der zionistischen Finanz sowie deren ekelerregenden Voelkermord Aktionen befreien.“
[ZJD_Gaza2009_624/816_ano_001]

Es werden auffällig oft Begründungszusammenhänge konstruiert, die den Holocaust rechtfertigen, indem Juden sowohl vergangenheits- als auch gegenwartsbezogen als globale Gefahr und Unheil dargestellt werden:
„Ja, ich glaube dass damals vor dem zweiten Weltkrieg die Juden die deutsche Bevölkerung vermutlich genauso bedrängt haben wie heute die Palästinenser. Die Juden verstanden es meisterhaft die Deutschen von Damals auszunützen und ihr Vertrauen zu missbrauchen.“ [ZJD_Gaza2009_20/816_ano_001]

Hitler wird in mehreren Schreiben als „noch viel zu human im Umgang mit euch jüdischen Verbrechern“ beschrieben. Für den Nahostkonflikt wird er als Autorität und Visionär angeführt:

(12) „Der Führer hätte so etwas nicht durchgehen lassen; da wären Plünderer, Vandalen und sonstige Volksschädlinge sofort erschossen worden, eben weil er Humanist war.“ [ZJD_Gaza2009_423/816_ano_001]

(13) „Ihr werdet die gleiche Behandlung bekommen die ihr den Palestinensern angedeihen laesst, da gibt es keine vertun. ...Hitler wird eines Tages noch nachtraeglich den Friedensnobelpreis verliehen bekommen, bei dem was ihr alles so treibt.“[ZJD_Gaza2009_730/816_Pan_001] 
Gemäß der nationalsozialistischen Konzeptualisierung von Juden als voLKsSCHÄDLINGEN werden alle Juden kollektiv entwertet, und ihre Ermordung wird als erstrebenswert dargestellt:

(14) „Wer einen Juden erschlaegt tut ein gutes Werk und handelt sogar im Sinne Gottes. Juden sind einfach nur kriminelle Verbrecher...“

[ZJD_Gaza2009_577/816_ano_001]

Was im antisemitischen Diskurs des 19. und frühen 20. Jahrhunderts als „Judenproblem" und „Judenfrage“ diskutiert wurde, findet sich expressis verbis auch im aktuellen Diskurs angesprochen (s. auch die Beispiele in Kap. 10.3):
„P.S. Ich denke ich konnte etwas zur Judenfrage beitragen! Nicht 'Ent- Lösung' der Judenfrage befreit uns von der Sklaverei und Machtgehabe dieser Welt, sondern die End-Lösung: Wer tatsächlich Jude ist und wer nicht!“ [ZJD_15.04.2002_Fal_001]

Kohärenzbrüche und semantisch kontradiktorische Argumentationen sind typisch: Der Holocaust wird einerseits als „noch nicht zu Ende gebracht“ angeführt, andererseits als historische Tatsache komplett geleugnet:

$$
\begin{aligned}
& \text { „So wie es aussieht haben unsere Großvaeter doch nicht gruendlich } \\
& \text { genug gearbeitet! Der Tag der Abrechnung wird kommen-Sieg Heil und } \\
& \text { dann bekommt ihr euren Holocaust den es bis JETZT noch nie gegeben } \\
& \text { hat... was noch nicht ist kann ja noch werden!HEIL HITLER!“ } \\
& \text { [IBD_19.01.2004_ano_001] }
\end{aligned}
$$

Insgesamt allerdings finden sich unter den Schreiben erstaunlich wenige Holocaustleugnungen, obgleich gerade diese jahrzehntelang als typisches Kennzeichen von Rechtsextremen und Neonazis galten (vgl. Lichtenstein 1999 und BfV 2005). Wesentlich häufiger wird entweder Bedauern darüber ausgedrückt, dass noch Juden leben, oder Stolz, dass immerhin sechs Millionen umgebracht wurden:

$$
\begin{aligned}
& \text { „[...] bin stolz darauf der Welt } 6 \text { Mio jüdische Parasiten erspart zu haben(.) } \\
& \text { Schade das wir das Ungeziefer nicht ganz ausrotten konnten und es nun } \\
& \text { immer noch an der Gesundheit userer Enkel nagt(.)Bald fahren wieder } \\
& \text { Juden in Aussschwitz ein!“ [IBD_06.04.2012_ano_004] }
\end{aligned}
$$

Verweise auf das Weltjudentum und das internationale Finanzjudentum finden sich ebenso wie die generische Referenz auf alle Juden mittels des typisierenden Singulars: 
„Aber was macht der 'Jude’ denn jetzt mit den Palästinensern? ... hat der 'Jude' damals nichts gelernt?““ [ZJD_Gaza2009_271/816_ano_001]

Dabei wird die Legitimierung des Holocausts durch Verweis auf die „verbrecherischen und kriminellen Aktivitäten der Israelis“ im Nahostkonflikt gerechtfertigt. Vermischungen von vergangenheits- und gegenwartsbezogenen Aspekten dominieren:

\begin{abstract}
„ihr seid und bleib Weltverbrecherschweine ..ermordung des adolf eichmann. Eure dreckigen mörderschweine von mossad haben adolf eichmann entführt und von einem dreckigen judenverbrecherlumpengericht zum tode verurteilt,alleine dafür gehört ihr judenschweine ausgerottet. beleidigung des adolf Hitlers Ihr dreckigen Weltverbrecherschweine beleidigt unseren lieben adolf und dürft straffrei herumlaufen das ist eine frechheit. Ich fordere die Todesstrafe für jedes dreckschwein das adolf beleidigt hat. Adolf Hitler ist viel zu human mit euch jüdischen Weltverbrechern umgegangen. Für den überfall auf den Libanon gehört Ihr Jüdischen Weltverbrecherschweine Ausgerottet"
\end{abstract}

[ZJD_13.08.2006_Kam_001] ${ }^{9}$

Interessant sind in diesem Zusammenhang (zahlreiche) Schreiben, in denen Juden einerseits gemäß der nationalsozialistischen Ideologie als minderwertige Rasse klassifiziert, andererseits als neue Nationalsozialisten deklariert werden. Logisch-konzeptuell ergibt sich hier ein (für den antisemitischen Diskurs durchaus typischer) Widerspruch: Die Nationalsozialisten verstanden sich als höherwertige Herrenrasse und die Juden als Untermenschen. Diese Auffassung vertreten ideologisch auch die rechtsextremen Verfasser. Nationalsozialisten haben in ihrem Weltdeutungsmuster den Status von Helden und Vorbildern. Wenn sie dann aber „die nicht-arischen Juden“ mit den ,arischen deutschen Nazis“ gleichsetzen, etablieren sie eine eklatante semantische Kontradiktion:

„Ihr seid die neuen NAZIS, ... einfach niedere Rasse!!!!!!!!!!!!!!!!!!!!!!!“ [IBD_25.10.2006_ano_001]

(21) „Ihr dreckigen verkommenen Juden-Nazis kotzt uns nur noch an!“ [ZJD_11.03.2007_Mon_001]

9 Fettdruck und Unterstreichung entsprechen dem Originaltext. 
$\mathrm{Zu}$ erklären sind diese in sich widersprüchlichen und widersinnigen Aussagen über das persuasive Potenzial von NS-Vergleichen: Kein Ereignis der deutschen Geschichte ist im kollektiven Bewusstsein so präsent und emotional so aufgeladen wie die NS-Zeit. Keine andere Epoche steht so sehr für Inhumanität und Monstrosität. Die Nationalsozialisten sind im modernen Bewusstsein der Nachkriegszeit Exponenten für die größtmöglichen Verbrechen an der Menschheit. Diese im gesellschaftlichen Bewusstsein verankerte und offiziell wie öffentlich vermittelte Bewertung wird daher auf die Juden und Israelis übertragen, um diese so intensiv wie möglich zu diskreditieren. Diese Funktion hat Priorität. Dass dabei ein konzeptueller Bruch mit der eigenen Ideologie zustande kommt, der die eigentlichen Identifikationsfiguren nun losgelöst vom nationalsozialistischen Weltbild aus der Perspektive der an sich abgelehnten demokratischen Gemeinschaft präsentiert, wird entweder gar nicht bemerkt oder in Kauf genommen. Widersprüche, Tautologien und Trugschlüsse sind aber nicht nur in den Texten von Neonazis und Rechtsextremisten anzutreffen, sondern sind generell ein charakteristisches Merkmal antisemitischer Texte (s. hierzu Kap. 9.4.2).

Der Bezug auf Hitler wird in vielen Schreiben im Rahmen von pseudo-kausal ausgerichteten Legitimierungen für die Verdammnis von Juden vollzogen:

„GHitler hatte doch recht !!!!!!!!!!!!!!!!!!!!!!!!!!!!!!!!!!!!!!!!!!!!!!!!!!!!!!!!!!!!!!!!!! !!!!!!!!!!!!!!!!!!!!!!!!!“ [ZJD_20.09.2007_ano_001]

(23) „Zutiefst erschüttert lese ich heute dass von der israelischen Armee, nach dem Krieg, Streubomben auf den Südlibanon geworfen wurden. In den letzten Wochen habe ich Hitler begriffen warum er die Juden ausrotten wollte! Ab heute sind die Juden auch meine Gegner,am besten ihr verschwindet als erstes aus Deutschland.Solche Kriegsverbrecher sind bei uns nicht erwünscht.“[ZJD_01.09.2006_Sch_002]

Der Verfasser von (23), der sich selbst als „nicht rassistisch“ und „kein Neonazi“, sondern als „linksliberaler Bürger“ beschreibt, sieht in der Gewalt im Nahostkonflikt einen Grund, alle Juden kollektiv als Kriegsverbrecher zu verurteilen und zugleich die Mordpolitik Hitlers zu rechtfertigen. Solche Rechtfertigungsmuster sind jedoch insgesamt bei gebildeten und linksliberalen Schreibern selten anzutreffen. Vielmehr distanzieren sich die meisten Verfasser aus der Mitte ausdrücklich von den Taten und der Ideologie der Nationalsozialisten. Allerdings greifen viele von ihnen dann mit NS-Vergleichen bezüglich des Nahostkonflikts auf rhetorische Brachialformen der irrealen Diskreditierung zurück und lassen eine extreme Feindbildrhetorik erkennen, wie in (24) und (25), den E-Mails von 
zwei sich selbst als „linke Stammwähler“ bzw. „links stehend“ bezeichnenden Akademikern, ${ }^{10}$ (24) von einem promovierten Münchner:

(24) „Das Israel von 2006 ist der faschistische Kettenhund der us-amerikanischen Rohstoffinteressen: so brutal, so inhuman und so entfesselt wie Hitlerdeutschland.“[ZJD_01.09.2006_Haf_001]

(25) „Der Krueppel- und Unrechtsstaat Israel ist die Fortsetzung der kranken Gedanken von NAZI Deutschland ...!“[IBD_15.06.2009_ano_001]

Immerhin 32 Prozent der Verfasser ${ }^{11}$ aus der Mitte (und acht Prozent aller gebildeten und hochgebildeten Schreiber) benutzen explizit wie in (26) und (27) oder implizit wie in (28) NS-Vergleiche:

(26) „Israel macht nichts anderes wie es HITLER gemacht hat !“ [ZJD_04.05.2007_Hof_001]

„IHR SEID JA FAST SCHLIMMER ALS DIE NAZIS FRÜHER!“ [IBD_29.06.2006_Mue_001]

(28) „Werter Zentralrat, ich bitte sie, ihren israelischen Brüdern und Schwestern für die Ermordung palästinensischer Kinder, Frauen und Alten die herzlichsten Kampfesgrüße zu übermitteln - hat Israel es nun doch geschafft, das Neveau des faschistischen Deutschland zu erreichen.“ [ZJD_Gaza2009_672/816_Nie_001]

10 Eine lexikalische Auffälligkeit ist hier zu beobachten: Während rechte Schreiber bei NSVergleichen das Wort nationalsozialistisch benutzen, verwenden Linke fast ausschließlich faschistisch; statt Holocaust bezeichnen sie die Militäraktionen Israels als ethnische Säuberungen. Rechtsextreme kodieren Verschwörungstheorien als jüdisch, Linksextreme als zionistisch. Vgl. hierzu auch die anti-israelischen Texte in der Roten Fahne, in der z. B. der Tod Barschels oder die Affäre um Dominique Strauss-Kahn als von Zionisten initiierte Ereignisse dargestellt werden.

11 Zwischen 2002 und 2007 benutzten nur 10,9 Prozent der Schreiber aus der Mitte explizite NS-Vergleiche: D. h. Juden und/oder Israelis werden mit Personen und Handlungen aus der NS-Zeit gleichgesetzt oder verglichen. Ab 2008 ist eine deutliche Zunahme zu verzeichnen: Repräsentative Stichproben innerhalb des neuen Materials bis Ende 2011 zeigen einen Prozentsatz von über 30 Prozent. Auch im Internet sind seit 2008/2009 verstärkt NS-Vergleiche bei der Diffamierung Israels zu sehen. 
Während NS-Vergleiche im öffentlichen Kommunikationsraum ${ }^{12}$ vor allem persuasiv benutzt werden, um die Aufmerksamkeit für die Äußerung zu erhöhen und Kritik an bestimmten Sachverhalten zu intensivieren, haben sie im antisemitischen Diskurs die Funktion, den bzw. die Verglichenen zu diffamieren:

\begin{abstract}
„Heute geht der weitaus größte und blutigste Teil der Gewalt von Israel aus, dabei handelt es sich mit Sicherheit um das größte Verbrechen der Gegenwart. Noch dazu, wo diese Gewalt im wesentlichen dazu dient, die völkerrechswidrige Schaffung von neuem Lebensraum für das auserwählte Volk zu sichern. (Auch hier drängen sich schlimme Parallälitäten auf!)“[ZJD_09.11.2006_Mei_001]
\end{abstract}

Nicht nur Israelis, auch die Vorstandsmitglieder des Zentralrats der Juden in Deutschland werden von akademischen Schreibern (insbesondere von sich selbst als „Friedensaktivisten“ bezeichnenden und links orientierten Verfassern) ${ }^{13}$ in Analogie zu Nazi-Größen gesetzt:

(30) „Die damaligen deutschen Machthaber wie Hitler, Göbbels usw. würden wohlehrfurchtsvoll zu Ihnen und Ihren Methoden aufblicken.“ [IBD_03.03.2006_Bur_001]

(31) „Wir hatten auch einen Herrn Dr. G... in unserer Historie und können deshalb Demagogie entsprechend werten. Mäßigen Sie sich, das ist besser für Ihre Sache.“ [ZJD_18.07.2006_Mue_001]

Israelische Militäraktionen werden mit dem staatlich angeordneten Massenmord an den Juden verglichen, Ramallah z. B. als zweites Warschauer Ghetto und Gaza als $K Z$ referenzialisiert, der Holocaust wird dadurch relativiert, die Einzigartigkeit der Verbrechen nivelliert und zugleich der Staat Israel drastisch dämonisiert. Teilweise werden die intertextuellen Verweise auf die nationalsozialistischen Vorlagen mittels Anführungszeichen als solche explizit markiert, ein Indikator, dass die Verfasser mit vollem Bewusstsein und intentional auf solche Sprachgebrauchsmuster zurückgreifen:

12 Zur Semantik und Pragmatik von NS-Vergleichen im öffentlichen Diskurs s. Schwarz-Friesel (2007: 195 ff.) und Eitz/Stötzel (2007: 312 f.).

13 Es gibt im Korpus eine Reihe von E-Mails, die von Mitgliedern der Partei Die Linke geschickt wurden. Diese benutzen im Vergleich zu anderen linksorientierten Schreibern signifikant häufiger explizite NS-Vergleiche. 
(32) „Ich bin sicher, Israel findet auch noch eine 'Endlösung' für die Palästinänser, was?““[IBD_28.06.2006_Hop_001]

(33) „Die israelische Siedlungspolitik in besetzen Gebieten kommt doch der Politik eines ‘Volkes ohne Raum' sehr nahe, und jetzt war in der militärischen Auseinandersetzung so etwas wie 'bis zum Endsieg' zu hören.“ [ZJD_Gaza2009_186/816_Sta_001]

Aber auch nonverbale Elemente werden benutzt; dazu zählen neben Karikaturen (die den Bildern im Stürmer oft frappierend ähneln) auch graphische Verschmelzungen von Davidstern und Hakenkreuz (also nonverbale symbolische NS-Vergleiche). Die Brisanz und die Unangemessenheit solcher Gleichsetzungen sind auch vielen Akademikern ${ }^{14}$ nicht bewusst bzw. werden gebilligt:

\begin{abstract}
„Das vorgehen Israels finde ich unbegreiflich.Im Fernsehen sah ich Bilder einer Demonstration gegen den Krieg (Malmö/Schweden) und ein Plakat Davidstern = Hakenkreuz. Ich finde über diesen Vergleich sollte wirklich intensiv nachgedacht werden.“[ZJD_29.07.2006_Pet_001]
\end{abstract}

Mit den gegen Israel (und zum Teil gegen alle Juden) gerichteten Boykottaufforderungen (die eher selten von rechtsextremen, dafür aber signifikant oft von linken und linksextremen Schreibern verbalisiert werden), die zumeist strukturidentisch mit den Appellen der Nationalsozialisten sind, greifen die Verfasser auf Strategien zurück, die als „Strafmaßnahmen“ bzw. „notwendige Handlungen“ deklariert werden, Juden bzw. Israelis aber kollektiv betreffen und damit genau dem ideologischen Anliegen der Nazis entsprechen.

$$
\begin{aligned}
& \text { „Deutsche, kauft keine israelischen Früchte.“[ZJD_10.08.2006_Neu_001] } \\
& \text { „Boykott zionistischer Waren!!“ [ZJD_07.05.2007_Nom_001] } \\
& \text { „Ich werde alles Jüdische ab sofort boykottieren! So wehre ich mich } \\
& \text { gegen die Unrechstpolitik Israels.“ [IBD_03.07.2008_Lam_001] }
\end{aligned}
$$

14 Unterzeichner und Befürworter von Boykottaufrufen verwahren sich ebenfalls stets energisch gegen den Vorwurf, dass ihre Aktivitäten eine judenfeindliche Tradition aus der NSZeit fortführen. 
Die Phrase aus der NS-Zeit, ${ }^{15}$ man müsse sich „gegen Juden zur Wehr setzen“ " ${ }^{16}$ wird in diesem Zusammenhang ebenfalls häufig artikuliert, entweder in der lexikalischen Variation, bei der das Objekt der Abwehr durch das Lexem Israel ausgedrückt wird, s. (37), oder wörtlich, wie in (38), aus dem Brief eines promovierten Akademikers aus Berlin:

„Ein Grund mehr mich in diesem Brief zumindest verbal gegen Juden zur Wehr zu setzen.“[ZJD_30.11.2006_Gel_001]

Der im Korpus zu konstatierende inflationäre Gebrauch der Lexeme Holocaust, Endlösung und Konzentrationslager zeigt eine zunehmende Bedeutungserweiterung ${ }^{17}$ dieser Ausdrücke in judenfeindlichen Texten: Die ursprünglichen Bedeutungen dieser Wörter werden durch den dekontextualisierenden, ahistorischen Gebrauch hinsichtlich ihrer Referenzfunktion verschoben. Sie referieren in den Texten nicht mehr auf die unikalen historischen Ereignisse und Stätten des Völkermordes, sondern werden als Diffamierungsmittel gegenüber Juden oder Israelis benutzt. So spiegelt sich die kommunikative Strategie der Täter-Opfer-Umkehr mit ihrer de-realisierenden Wirkung auch auf der verbalen Mikrostrukturebene wider.

\subsection{Lexemanalysen zu Frechheit/frech und hetzen/Hetze}

Die Stichproben-Analyse eines Teilkorpus von 1.034 digital vorhandenen Zuschriften an den Zentralrat der Juden in Deutschland und von 2.000 der digital vorhandenen Zuschriften ${ }^{18}$ im Botschaftskorpus ergab, dass die Lexeme frech

15 In vielen Texten wird auch die Phrase von der „gleichgeschalteten Presse“ benutzt, um auf das angebliche Meinungsdiktat zu verweisen. Vgl. hierzu auch Günter Grass' Reaktion auf die Kritik an seinem anti-israelischen Gedicht, in der er von einer „fast wie gleichgeschalteten Presse" sprach (vgl. Kap. 7).

16 Vgl. etwa: „Deutsche, wehrt euch, kauft nicht bei Juden!“(Berlin, 1933, vgl. Königseder 2011: 61)

17 Diese Tendenz ist nicht nur im judenfeindlichen Diskurs zu beobachten: Der inflationäre Gebrauch von NS-Vergleichen (vgl. Äußerungen wie Der Holocaust auf Ihrem Teller; BabyCaust; Holocaust an den Palästinensern) im öffentlichen Kommunikationsraum kann dazu führen, dass sich das Bewusstsein für die historische Verankerung und die Einmaligkeit dieses Verbrechens und sich dann auch die Bedeutung des Wortes Holocaust dekontextualisiert verändert, d. h. das Referenzpotenzial größer wird, es also zu einer Bedeutungserweiterung kommt der Art 'Holocaust = Verbrechen'. Dies ginge zwangsläufig mit einer mentalen Relativierung der Verbrechen an den Juden in der NS-Zeit einher (vgl. hierzu auch Soric 2005). 1838 Zuschriften, in denen die Lexeme in Kollokationen vorkamen, wurden qualitativ näher analysiert, um die eventuelle Einbettung dieses Sprachgebrauchs in antisemitische 
und Frechheit ${ }^{19}$ signifikant oft in der Verbindung mit den Lexemen Jude/jüdisch verbalisiert werden. Die Frage, die sich hier stellt, ist, ob es sich lediglich um die Reproduktion von bekannten Floskeln aus der NS-Zeit handelt ${ }^{20}$ oder ob es in den Texten, die solche Phrasen enthalten, auch semantisch-konzeptuell Korrelationen mit dem Gedankengut der Nationalsozialisten gibt. Im Sprachgebrauch des Nationalsozialismus findet sich die Negativbewertung von Juden und jüdischem Verhalten mittels des Lexems frech in verschiedenen Varianten in vielen Reden und Schriften, wie z. B. in den Phrasen „echt judenhaft frech“ (Hitler 1934: 314) oder „eine wahrhaft jüdische Frechheit“ (Hitler 1934: 248) bzw. in den gegenüber Juden geäußerten Vorwürfen, wie „seine [= des Juden, d. Verf.] Wucherzinsen erregen endlich Widerstand, seine zunehmende sonstige Frechheit aber Empörung“ (Hitler 1934: 339). Juden werden somit generell als UNVERFROREN, DREIST, SICH NICHT IN DIE GESELLSCHAFT EINFÜGEND, SICH GEGEN DAS GEMEINWOHL RICHTEND konzeptualisiert, wie es sich in den Stereotypen JUDEN SIND AMORALISCH, JUDEN SIND STÖRENFRIEDE, JUDEN SIND ZERSETZER manifestiert. ${ }^{21}$

Argumentationsmuster zu überprüfen. Die Textanalyse zeigte, dass das Merkmal 'frech' als typisch jüdisch angesehen wird und so ein gängiges Klischee darstellt. Der nachhaltige Einfluss der NS-Propaganda ist hier nicht von der Hand zu weisen.

19 Im aktuellen deutschen Sprachgebrauch existiert eine pejorative Lesart für das Adjektiv frech, d. h. im negativen Sinne von dreist, wenn jemand also ohne Achtung und Respekt vor anderen ist und als 'unverschämt' bzw. 'asozial' charakterisiert wird. Als Synonyme gelten dreist, impertinent, pampig, patzig, schnippisch, schnoddrig, unartig, ungezogen, unverfroren, unverschämt, vorlaut, vorwitzig (vgl. Duden $\left.{ }^{3} 2002\right)$. Über die Erklärungen des Dudens hinausgehend verweist der Lexikoneintrag der freien Enzyklopädie Wikipedia als Informationsportal von der und für die Allgemeinheit darauf (sie zeigt somit auch das allgemeine Bedeutungsverständnis der Lexeme in der Alltagssprache auf), dass es sich bei der Prädikation als 'frech' um eine moralische Abwertung bzw. Negativevaluierung handelt. Des Weiteren wird ein Querverweis zum Lexem Chuzpe aus dem Jiddischen angegeben. Der Eintrag auf Wikipedia belegt, dass im gegenwärtigen Sprachgebrauch ebenfalls eine Assoziation zwischen frech/Frechheit und Juden/Jüdischem besteht (vgl. http://de.wikipedia.org/wiki/ Frechheit, letzter Zugriff am 02.09.2012).

20 Aufgrund der jahrzehntelangen Thematisierung und Erörterung der NS-Ideologie in Bildungseinrichtungen und in den Massenmedien ist mit einem solchen Effekt durchaus zu rechnen. Besonders häufig besprochene und analysierte Reden und Schriften von NS-Größen sind als Teil des kollektiven Wissens zu betrachten und haben daher im Langzeitgedächtnis den Status 'schnell aktivierbar'. Dass lexikalische Einheiten unterschiedliche Aktivierungsgrade aufweisen, ist in der Psycho- und Kognitionslinguistik intensiv erforscht worden (s. z. B. Aitchison ${ }^{4} 2012$ und Schwarz ${ }^{3}$ 2008: 228).

21 Gegenwärtig findet sich diese Konzeptualisierung von Juden auch explizit in rechtsextremistischer Propaganda, beispielsweise wird in einem Liedtext der Gruppe Weißer Arischer Widerstand auf diese alte antisemitische Verbalisierung zurückgegriffen: „Sollen freche Juden uns're Herren sein? Wo sitzen Deutschlands schlimmste Feinde? In der jüdischen Gemeinde!“ (DÖW, Dokumentationsarchiv des österreichischen Widerstandes, verfügbar unter: http:// 
In den Zuschriften an den Zentralrat der Juden in Deutschland finden sich die Lexeme frech und Frechheit dabei weitaus häufiger als in den Zuschriften an die Israelische Botschaft in Deutschland. Dieser Unterschied der Verwendungshäufigkeit weist darauf hin, dass auch aktuell noch diese Negativevaluierung und moralische Abwertung von Juden und ihren Handlungsweisen stärker habitualisiert und als usueller im mentalen Lexikon der Sprachbenutzer abgespeichert ist als in der Kollokation freche Israelis. Allerdings zeichnet sich hier bereits eine Veränderung ab: Kommen Verbalisierungen wie „die frechen Israelis“ [ZJD_24.06.2002_Mau_001] bis 2005 nur vereinzelt in den Texten vor, ist ab 2006 ein deutlicher Anstieg in der Verwendungshäufigkeit zu konstatieren. Analog zur Konstruktion die frechen Juden sind vermehrt Referenzialisierungen der Art die frechen Israelis zu registrieren. Hier drückt sich die konzeptuelle Verschmelzung von Juden und Israelis aus, wobei auf das Konzept ISRAELIS antisemitische Stereotype übertragen werden.

Diese spezifische Form des Verbal-Antisemitismus findet sich dabei in den Zuschriften an den ZJD und die IBD jedoch nicht primär bei Schreibern aus der rechten oder rechtsextremen Ecke, wie zu vermuten wäre, sondern bei Verfassern, die der gesellschaftlichen Mitte zuzuordnen sind. ${ }^{22}$ So beschwert sich ein Herr in einer umfangreichen E-Mail an den Zentralrat über Michel Friedman wie folgt:
„Sehen Sie, genau das ist [...] eine nicht mehr zu überbietende jüdische Frechheit und Unverfrorenheit. [...] Immer wieder beschweren sich bei mir am Telefon und im Internet Freunde, Bekannte und Geschäftsleute von überall bis nach Österreich und die Schweiz über das rotzfreche Auftreten dieses Herren [= Michel Friedman, d. Verf.].“[ZJD_30.07.2006_Ren_001]

Die semantische Generalisierung durch die Nominalphrase „jüdische Frechheit“ zeigt, dass die Verächtlichmachung sich nicht allein auf die Person Michel Friedman und sein Verhalten als Moderator bezieht, sondern es wird dadurch allen Juden generell ein moralisch diskreditierendes Verhalten unterstellt. Bei einer solchen Generalisierung ist die Eigenschaft 'frech' in ihrer negativevaluierenden Lesart inhärent an die Kategorie JüDIscH gekoppelt. Das Verhalten des Zentralrats wird in den analysierten E-Mails entweder in Verbindung mit dem Stereotyp

\footnotetext{
de.doew.braintrust.at/b163.html, letzter Zugriff am 02.09.2012).

22 Von den 38 einzeln und detailliert analysierten Zuschriften mit den Phrasen freche Juden bzw. jüdische Frechheit sind nur zwei als rechtsextrem und alle anderen politisch als von Verfassern aus der gesellschaftlichen Mitte produziert zu verorten. Bei diesen ist eigentlich mit einer erhöhten Sensibilität gegenüber NS-Stil und Feindbildrhetorik zu rechnen, was aber keineswegs der Fall ist.
} 
JUDEN NUTZEN DIE VERGANGENHEIT ZU IHREN FINANZIELLEN GUNSTEN AUS Oder mit dem Stereotyp des MEINUNGSDIKTATS als Ausdruck der unmoralischen Eigenschaften von Juden prädiziert.

(40) „Es ist eine Frechheit, wie Sie versuchen die BRD, 60 Jahre nach Ende des dritten Reiches, mit der Vergangenheit zu erpressen. In Zeiten knapper Kassen stellen Sie frech immer höhere Forderungen nach finanzieller Unterstützung.“[ZJD_12.08.2006_Gru_001]

(41) „Kommentare des zentralrates der Juden empfinde ich immer wieder als freche Einmischung und bösartige Provokation aber vielleicht bin ich ja für die Juden auch nur wieder so ein Nazi ( weil ich meine Meinung sage !!)“ [ZJD_Gaza2009_302/816_Wel_001]

In den Spezifizierungen der unterstellten Frechheit zeigen sich die stereotypen Konzeptualisierungen von Juden, die für die Textproduzenten durch die Mitglieder des Zentralrats pars pro toto personifiziert werden. Die Verfasser bedienen das Klischee, dass Juden die deutsche Verantwortung für die Vergangenheit finanziell und intellektuell ausnutzen würden, und greifen somit ein dominantes Nachkriegsstereotyp auf. Gleichzeitig wird das tradierte Stereotyp vom RACHSÜCHTIGEN und UNVERSÖHNLICHEN JUDEN kodiert.

Das Verhalten von Repräsentanten des Zentralrats wird auch dann als frech/ Frechheit bezeichnet, wenn diese sich kritisch über antisemitische und/oder antiisraelische Vorfälle äußern. So wird in einem Brief Paul Spiegel eine unrechtmäßige Einmischung vorgeworfen:

\begin{abstract}
„Das kann nicht sein, und es wird nicht so kommen, daß Sie in die deutsche Parteienlandschaft herein reden. Und es ist eine ausgesprochene Frechheit von Ihnen, einen, wenn auch Populisten, Herrn Möllemann, des Antisemitismus zu zeihen. [...] Darf ich Sie daran erinnern, wie sie es immer treiben, die Juden?“ [ZJD_18.06.2002_Ser_001]
\end{abstract}

Die Argumentation von (42) baut auf dem antisemitischen Stereotyp JUDEN SIND KEINE DEUTSCHEN auf. Das Recht auf freie Meinungsäußerung wird Paul Spiegel explizit und ausdrücklich abgesprochen. Dass sich Spiegel - ein Jude - kritisch gegenüber dem deutschen Politiker Jürgen Möllemann äußert, wird als ungehörige und nicht hinzunehmende Frechheit prädiziert. Mit dieser Entwertung geht darüber hinaus eine generelle Abwertung von Juden einher, die sich insbesondere in der anschließenden rhetorischen Frage zeigt. Über die Gradpartikel immer und 
die kollektive Generalisierung mittels der generischen Nominalphrase die Juden wird Paul Spiegels Verhalten pauschal als typisch jüdisch deklariert.

Eine konzeptuelle Verschmelzung von judeophoben und anti-israelischen Stereotypen ist in der Zuschrift eines Herrn aus Aschaffenburg zu finden:

„Was derzeit Scharon ausführt mit seinen Gefolge, das ist eine jüdische Frechheit, die zum Himmel schreit.“ [ZJD_06.04.2002_Klo_001]

Über die auf Ariel Scharon bezogene Phrase der jüdischen Frechheit wird zum einen eine Gleichsetzung von ISRAELISCH und JÜDISCH ausgedrückt, zum anderen die moralische Unverfrorenheit aller Juden prädiziert.

Eine weitere lexikalische Auffälligkeit im Korpus ist die häufige Verwendung der Lexeme hetzen und Hetze bzw. Kompositionen auf der Basis von Hetz-. ${ }^{23}$ Mit dieser Lexemverwendung geht eine Negativevaluierung einher über den Vorwurf, unzulässige Propaganda bis hin $\mathrm{zu}$ strafrechtlich relevanter Volksverhetzung $\mathrm{zu}$ betreiben. Werden Juden und/oder Israelis als Hetzer bezeichnet, ${ }^{24}$ werden häufig auch die Stereotype KRITIKTABU und JÜDISCHER EINFLUSS bezogen auf die Meinungsfreiheit in der deutschen Gesellschaft ausgedrückt. Beispiel (44) verdeutlicht, dass eine antisemitische Konzeptualisierung von Juden, die eng mit den Lexemen Hetze/hetzen verbunden ist, gleichzeitig an eine negativ evaluierende und emotional abwertende Bedeutung gekoppelt ist:

(44) „Weshalb, liebe Frau Knobloch, hetzen Sie in dieser für sie typischen Weise gegen deutsche Bischöfe? Fehlt es Ihnen an der nötigen Bildung? Nein? Dann ist's also doch nur blindzionistischer Deutschenhass?“ [ZJD_11.03.2007_Ise_001]

23 Im aktuellen Sprachgebrauch werden die Lexeme hetzen und Hetze zum einen in der Bedeutung von 'sich sehr beeilen' bzw. 'überstürzte' oder 'übertriebene Eile' verwendet (Duden ${ }^{3}$ 2002), wobei dieser Bedeutungsaspekt im untersuchten Korpus keine Rolle spielt. Zum anderen werden diese Lexeme für das Jagen oder Verfolgen ursprünglich von Tieren im Sinne einer Treibjagd, aber auch in Bezug auf Menschen verwendet. In Erweiterung dieser Bedeutung werden 'unsachliche, gehässige, verunglimpfende Äußerungen und Handlungen, die Hassgefühle, feindselige Stimmungen und Emotionen gegen jmdn., etwas erzeugen' (Duden ${ }^{3} 2002$ ) als Hetze beschrieben. Im nationalsozialistischen Sprachgebrauch wurden diese Lexeme bzw. Determinativkomposita wie Hetzblatt, -artikel, -schrift, -rede als Propagandawörter (Paul ${ }^{10} 2002$ : 471) zur Diffamierung von Juden und politischen Gegnern gebraucht.

24 Im Zentralrats-Korpus werden als hetzerisch Handelnde bei ca. zwei Drittel der Zuschriften und im Botschafts-Korpus bei ca. einem Drittel der Zuschriften Juden und/oder Israelis 
Bei der Textproduzentin ist das Konzept JUDEN inhärent mit dem schon für die NS-Zeit typischen Stereotyp von JUDEN ALS HETZER verknüpft, denn die ZJD-Vorsitzende Charlotte Knobloch hetze in der „für sie typischen Weise“. Das Personalpronomen sie wird hier in der dritten Person Plural und nicht in der zweiten Person Plural als persönliche Anredeform verwendet, sodass nicht eine für den Menschen Knobloch typische, sondern für alle Juden typische Verhaltensweise kritisiert wird. Trotz der in Frageform gehaltenen Äußerungen sieht die Verfasserin die Ursache für Knoblochs Kritik an den Äußerungen der deutschen Bischöfe in Ramallah eindeutig in einem den Juden über die Kommentaradverbien „also doch nur“ pauschal unterstellten „blindzionistischen Deutschenhass“ - es ist somit nicht das Außergewöhnliche, was mangelnde Bildung wäre, sondern das Typische, der angeblich jüdische Hass auf Deutsche, der Knobloch zu ihrer Kritik motiviert. Dieser Konzeptualisierung liegt das aktuelle Stereotyp JUDEN SIND UNVERSÖHNLICH zugrunde, welches zu der pseudo-kausalen Argumentationskette führt: 'Juden hetzen gegen Deutsche, weil sie unversöhnlich sind'. Indirekt tritt der Vorwurf zutage, dass Juden die Vergangenheit nicht ruhen lassen können und somit dem Wunsch der Nachkommen der Täter nach Vergessen und einem Schlussstrich unter die NS-Zeit nicht nachgeben.

In den Zuschriften, bei denen mit der Verwendung der Lexeme Hetze/hetzen antisemitische Konzeptualisierungen einhergehen, findet oftmals eine Verknüpfung von nationalsozialistischem Sprachgebrauch und kollektiven Schuldzuweisungen statt.

„Ihr habt dafür gesorgt, daß wir in Europa die Revolution und die Weltkriege bekamen. Weltweit 200.000 Millionen Tote durch Krieg, Revolutionen, Bürgerkriege gehen auf das Konto eurer Hetzer und eurer kommunistisch, kapitalistischen menschenverachtenden Lügenideologie. Euer religiös bemäntelter Rassismus ist ekelhaft!“ [IBD_19.07.2006_Bec_001]

Argumentationsmuster des Nationalsozialismus ${ }^{25}$ werden auf aktuelle Ereignisse des Nahostkonflikts übertragen: Juden werden für die historisch-gesellschaftlichen und wirtschaftlichen Entwicklungen der Moderne sowie für die Gräuel der Weltkriege verantwortlich gemacht. Auf dieser Vorstellung von der prinzipiellen

benannt. Gleichzeitig enthalten diese Zuschriften antisemitische Konzeptualisierungen. Knapp vier Fünftel der Verfasser solcher Texte entstammen der gesellschaftlichen Mitte.

25 So schreibt Hitler (1934: 162): „Tatsächlich waren es auch in erster Linie Juden und Marxisten, die hier mit allen Mitteln zum Kriege zwischen den zwei Staaten schürten und hetzten.“ Oder (Hitler 1934: 702): „So ist der Jude heute der große Hetzer zur restlosen Zerstörung Deutschlands.“ 
Schlechtigkeit und Macht der Juden aufbauend wird das heutige Verhalten Israels als hinterhältig und verabscheuungswürdig konzeptualisiert (s. Kap. 7).

Das Kompositum Hetzkampagne wird in vielen Texten gekoppelt an das Stereotyp des KRITIKTABUS artikuliert, wie in der E-Mail eines „überzeugten Katholiken“ und „Gutmenschen“:

(46) „Wenige haben dies versucht und wurden [...] daraufhin einer regelrechten Hetzkampanie ausgesetzt, wie dem damaligen Politiker Möllemann, der sicher nicht immer richtig gehandelt, jedoch den Mut aufgebracht hat, etwas zu sagen! Oder sind wir zum Schweigen verurteilt?“ [ZJD_27.07.2006_Stu_001]

Implizit zeigt sich hierin auch eine Antisemitismus-Abwehr bzw. die Leugnung und Marginalisierung von judenfeindlichen Äußerungen. Besonders oft werden auf Israel bezogene Solidaritätsaufrufe des ZJD als „freche Propaganda“, „Hetze“ und „Unverschämtheit“ bezeichnet und als kriminell eingestuft:

„Wenn ich nochmals 'so eine’ Presseerklärung [= Aufruf des Zentralrats zur Solidarität mit Israel, d. Verf.] höre werde ich ab da die naechsten Jahre nur noch Kreuze bei der NPD machen. Das Resultat wird mir dann egal sein. So, und nun wuensche ich euch auch weiterhin viel Spaß bei der Volksverhetzung.“ [ZJD_30.07.2006_Ren_001]

Die angekündigte Bereitschaft und Androhung des Verfassers von (47), eine rechtsextreme Partei zu wählen, deren antisemitische Positionen hinlänglich bekannt sind, wenn der Zentralrat zukünftig seine Haltung nicht verändert, impliziert, es bestehe ein kausaler Zusammenhang zwischen dem Verhalten von Juden und Antisemitismus.

\section{Fazit}

Die Analyse der Texte gibt Aufschluss, in welcher Weise im Gebrauch der deutschen Gegenwartssprache formal und inhaltlich auf die Ideologie der Nationalsozialisten Bezug genommen wird und wie sich die Vergangenheitsbewältigung im judenfeindlichen Diskurs sprachlich artikuliert. Werden Wörter, Phrasen und Sätze aus antisemitischen Schriften und Reden der NS-Zeit benutzt, korreliert dies zumeist auch mit der Verbalisierung judenfeindlichen Gedankenguts. Die Reproduktion von Versatzstücken aus der NS-Sprache geht also in der Regel auch mit der Kodierung antisemitischer Stereotype einher. Es handelt sich somit nicht 
nur um den unreflektierten Zugriff auf im Gedächtnis gespeicherte Floskeln, die aufgrund der Bekanntheit schnell und präferiert aktiviert werden. Vielmehr wird mit der Verwendung des Ideologievokabulars auch eine judenfeindliche Einstellung ausgedrückt. Während rechtsextremistische Textproduzenten in diesem Zusammenhang auch nahezu identisch die Konzepte des rassistischen und eliminatorischen Antisemitismus artikulieren und belegen, wie ideologisch geprägte Weltsicht und Sprachgebrauch im Rechtsextremismus ineinandergreifen, zeigt sich bei Verfassern aus der Mitte eine Loslösung vom rassistisch fundierten Antisemitismus bei gleichzeitiger Verschiebung zum anti-israelischen Antisemitismus. Wie bei der Artikulation der Stereotype zeichnen sich die Texte dabei allerdings durch eine Symbiose von gegenwarts- und vergangenheitsbezogenen Argumenten aus. Verfasser aus der Mitte projizieren sowohl tradierte als auch in der Nachkriegszeit entstandene Stereotype der Judenfeindschaft auf Israel.

Die kognitiven und emotionalen Auswirkungen der NS-Zeit und die sich daraus ergebende Vergangenheitsbewältigung spiegeln sich im judenfeindlichen Sprachgebrauch über alle Grenzen der politischen, sozialen und ideologischen Ausrichtung wider. Es lassen sich aber deutlich erkennbare gruppenspezifische Differenzen feststellen: Rechtsextreme rekurrieren sowohl formal als auch inhaltlich nahezu identisch auf nationalsozialistische Konzeptkategorien und Entwertungsmuster. Sie differenzieren nicht zwischen Juden und Israelis. Holocaustleugnungen, die jahrzehntelang ein charakteristisches Merkmal in der Argumentation von Rechtsextremen und Neonazis waren, finden sich selten. Stattdessen wird entweder bedauert, dass die Nationalsozialisten nicht effektiv genug waren und es noch Juden auf der Erde gibt. Oder der Holocaust wird als Vergleichsgröße angeführt, um die aktuell lebenden Juden in Israel zu diffamieren, indem die Militäraktionen Israels mit dem gezielten Massenmord in der NS-Zeit gleichgesetzt werden.

Linke und linksextreme Schreiber benutzen viele NS-Vergleiche in Bezug auf Israel, in denen sie jedoch die Sprachmuster des Nationalsozialismus verändern und ihrer ideologisch-politischen Einstellung gemäß anpassen. Verschwörungstheoretische Konstrukte werden über einen extremen Antizionismus ausgedrückt. Verfasser der Mitte kombinieren bei ihrer Erinnerungs- und Schuldabwehr vor allem Stereotype der klassischen Judenfeindschaft mit Klischees der Nachkriegszeit und benutzen die Strategie der Täter-Opfer-Umkehr projiziert auf Israel, indem sie vor allem implizite NS-Vergleiche kommunizieren.

Als Gemeinsamkeit ist bei allen Verfassern, die NS-Sprachgebrauchsmuster (identisch oder modifiziert) reproduzieren, zu konstatieren, dass sie auf judenfeindliche Konzeptualisierungen zurückgreifen und den Aspekt der Vergangenheitsbewältigung durch schuldzuweisende oder -abwehrende Verbalangriffe reduzieren und kanalisieren. 